\title{
- Survey of Trends in Electrotherapy in Gujarat
}

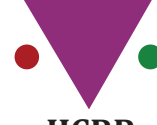

IJCRR

Section: Healthcare

ISI Impact Factor

(2019-20): 1.628

IC Value (2019): 90.81

$\operatorname{SJIF}(2020)=7.893$

\section{Gosai G' ${ }^{1}$, Dave $\mathbf{Y}^{\mathbf{1}}$, Parmar J², Chhatlani $\mathbf{R}^{\mathbf{1}}$}

'Assistant Professor, Faculty of Physiotherapy, Marwadi University, Rajkot, Gujarat, India; ${ }^{2}$ Principal, Faculty of Physiotherapy, Marwadi University, Rajkot, Gujarat, India.

\section{ABSTRACT}

Introduction: Electrotherapy has been a very important part of physiotherapy treatment, yet it is an area of debate and research that how it is being practised. Specifically use of Ultrasound, Short wave Diathermy and Traction (Cervical and Lumbar).

Aims: To find the trends in the use of Electrotherapy modalities in Gujarat.

Methodology: A questionnaire with a variety of questions on the application and frequency of prescribing modalities to patients was prepared and circulated online to 30 Physiotherapy Colleges in Gujarat.

Result: Data obtained from all colleges was analyzed by SPSS 24.0. The Means were utilized to observe the trends in the use of Electro-modalities. $(p<0.05)$

Conclusion: It was observed that all of the institutes were using Ultrasound (US) and Cervical Traction (CT) frequently. Majority is giving the US for 5-7 min, ignoring many aspects in deciding the dosage of it.CT is usually not differentiated with lesion site but given in neutral position. Short-wave Diathermy (SWD) is given according to available resources or in general about 10-15 min in the Co-planner method.

Key Words: Physiotherapy, Electro modalities, Ultrasound, Short-wave Diathermy, Lumbar Traction, Cervical Traction

\section{INTRODUCTION}

Electrotherapy is the main module of physiotherapy practice. Modalities like Cervical Traction (CT), Lumbar Traction (LT), Ultrasound (US) and Short-wave Diathermy (SWD) are frequently used in a physiotherapy clinic. ${ }^{1,2}$

Cervical traction is a modality of choice for many cervical dysfunctions. It applies to a wide range of problems from a sprain to fractures and dislocations of the cervical vertebrae.

Mainly there are 2 ways in which CT can be given

1. Static traction: Traction with a constant pull varying from 10 to $30 \mathrm{lbs}$ is applied for $20-25 \mathrm{~min}$. It is indicated in the presence of definite neurological signs, including radiating pain, not relieved by other conservative modes.

2. Intermittent traction: Traction with alternate phases of stretching (pull) and relaxation is the popular mode of traction. It produces the effects of massage on the muscular, ligamentous and capsular structures. It promotes circulation and reduces swelling, thereby reducing inflammation, spasm and pain. ${ }^{3}$

Lumbar Traction is also known as spinal traction. It is a popular modality in the management of Low Back Pain (LBP). Again, there are 2 main ways in which LT can be given

1. Sustained traction: In supine lying, the static traction with a tractive force of not less than $50 \%-60 \%$ of the bodyweight is applied for 20-30 min.

2. Intermittent traction: Alternate application of traction and its release is the most popular method of applying lumbar traction. It has a vascular massaging effect, and relaxation is produced in the tight soft tissues. The combination of alternating stretch and release promotes relaxation. It is well accepted by the patients as compared to sustained traction. ${ }^{4}$

In Ultrasound therapy, ultrasound energy is used to treat human tissue. The energy has applied to a transducer which

\section{Corresponding Author:}

Chhatlani R, Saraswati-Narayan, Silver Stone Society, Opposite Oscar Tower, 150 ft. Ring Road, Rajkot-360005, Rajkot, Gujarat, India. Phone: 9879878157; Email: rahul.chhatlanipt@gmail.com

ISSN: $2231-2196$ (Print)

Received: 09.01 .2021
ISSN: 0975-5241 (Online)

Revised: 26.02 .2021
Accepted: 17.04 .2021

Published: 12.09 .2021 
consists of a crystal, which vibrates to produce energy. The US is a form of acoustic vibrations propagating in the form of longitudinal compression waves at frequencies too high to be heard by human ears.

There are various means by which the US can be given to the human tissue.

1. Direct contact with Gel: On a regular skin surface, a coupling medium is applied to eliminate air between the skin and the transducer and transmit the ultrasound beam to the tissues. The transducer head is moved in small concentric circles over the skin, keeping the metal front plate in full contact with the surface.

2. Water Bag: irregular bony surfaces, a rubber bag filled with degassed water is used. A coupling medium is placed between the rubber bag and the skin, and between the rubber bag and transducer head. The treatment head is moved over this rubber bag. The only disadvantage is of attenuation of ultrasound, as it has to cross many interfaces.

3. Water Bath: A water bath is filled with de-gassed water if possible. Ordinary water presents a problem that gas bubbles dissociate out from the water. These bubbles accumulate on the skin and transducer head, and so reflect the ultrasound beam. Then these bubbles must be wiped from these surfaces regularly. ${ }^{5}$

Short wave Diathermy is a deep heating modality. Radio waves in the short-wave band have a frequency in the range of $10 \mathrm{MHz}-100 \mathrm{MHz}$. The short-wave diathermy used widely utilise the frequency of $27.12 \mathrm{MHz}$ and wavelength greater than $11 \mathrm{~m}$.

There are further 3 methods of applying SWD to the patients:

1. Co-planar: It is also called the parallel method. The electrodes are placed side by side.

2. Contra-planar. It is also called through and through the method. Pads are placed on either side of the joints. In this method, deeper tissues are heated. ${ }^{6}$

3. Crossfire Method: In this technique, half of the treatment is given with the placement of electrodes in one direction, i.e., medial or lateral aspect and another half is used with the placement of electrodes in other direction, i.e., anterior or posterior aspect.

This method is commonly used for the treatment of the knee joint, sinuses (frontal, maxillary and ethmoidal) and pelvic organs. $^{7}$

\section{Need of the study:}

Electrotherapy has been a very important part of physiotherapy treatment, yet it is an area of debate and research that how it is being practised. Choosing parameters and techniques for Electro Physical Agents (EPAs)is not simple. Although, when used inappropriately, EPAs can be ineffective. Clinical decision making is found to be dependent upon past experiences and the availability of resources. This study intends to find how US, LT, CT and SWD are used in educational institutes, as it is likely to affect future therapist's clinical decision making.

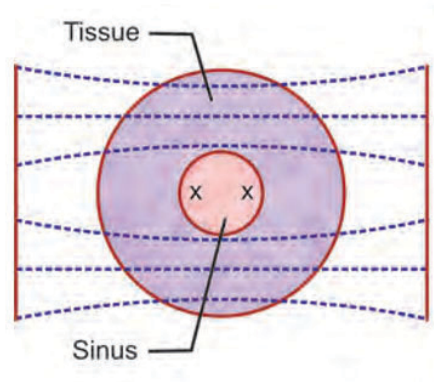

(a)

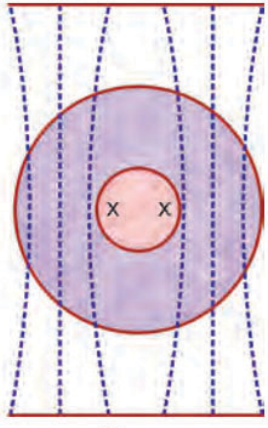

(b)
Figure 1: ShowsCrossfire Method.

\section{AIMS AND OBJECTIVES:}

Aim:

To find the trends in the use of Electrotherapy modalities in Gujarat

\section{Objective:}

To prepare and circulate survey questionnaire.

To obtain data from colleges in Gujarat regarding the survey.

To analyse the data obtained.

\section{METHODOLOGY:}

Study Design: A Cross-sectional Survey

Sample Size: 30 Colleges participated in the study

Sampling Method: Purposive Sampling

Study setting: Faculty of Physiotherapy, Marwadi University, Rajkot.

Study Duration: April 2020 to May 2020

\section{Method:}

A brief Questionnaire was prepared to understand the trends in Electrotherapy Modality usage among various colleges and their Out-Patient Departments/Clinics.

35 Colleges were approached in Gujarat out of which 31 Colleges responded with data. Out of 30 Colleges, data of one college was found to be incomplete hence 30 college data were included and analysed.

This Questionnaire is intended to know techniques used by physiotherapists across Gujarat regarding Electrotherapy 
modalities like Ultrasound, Traction (Lumbar and Cervical) and Short-wave diathermy.

The Questionnaire was prepared in Google Forms.

The Questionnaire was forwarded to all colleges in Gujarat through E-mail and consent was taken for not disclosing their data.

The data was then obtained through e-mail and analysed.

\section{RESULT}

Data were analysed using SPSS Version 24.0 and the $\mathrm{p}$ value was set to 0.05 . Means were calculated as a measure of central tendency for all data obtained.

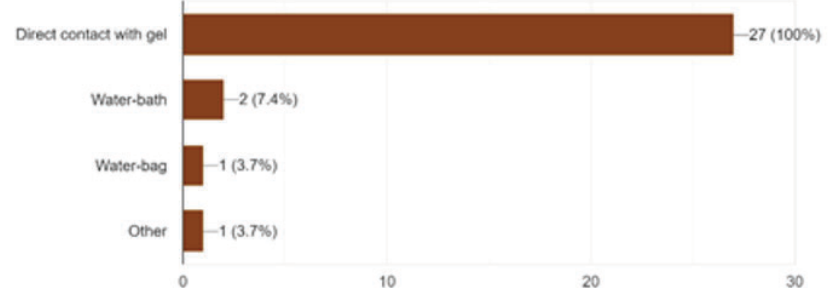

Figure 2: Use of Method of application of US.

It should be noted that the Questionnaire contained checkboxes for Use of the method of application of US and factors to decide the frequency of US, hence the total number of samples is more than $\mathrm{n}(=30)$.

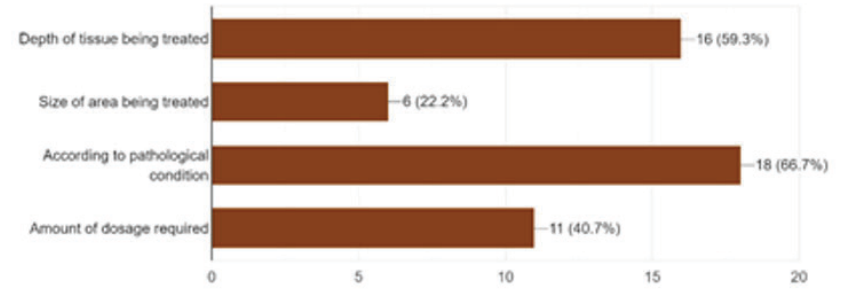

Figure 3: Factors to decide Frequency of US.

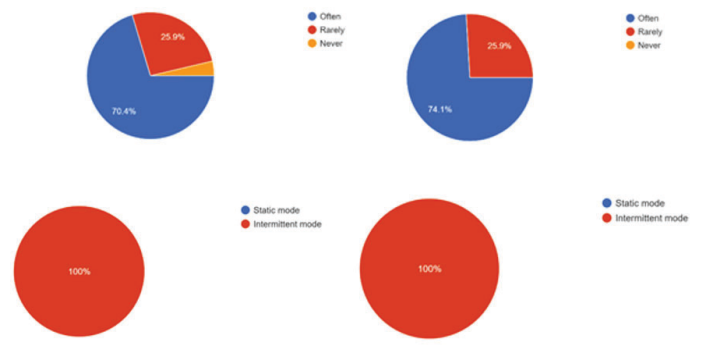

Figure 4: (a) How often CT is used in the clinic, (b) How often is LT used in the clinic, (c) Which method of CT is utilized and (d) Which method of LT is utilized.

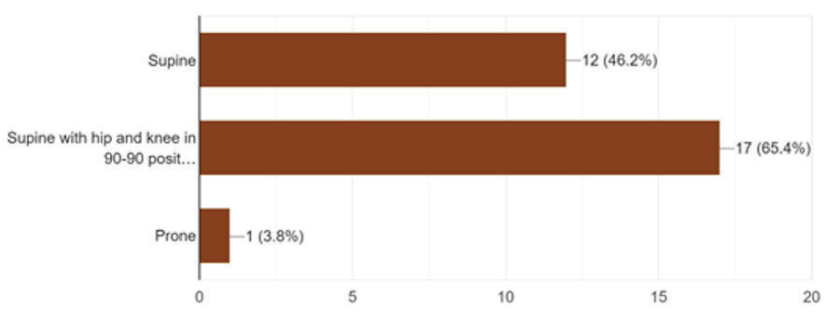

Figure 5: Position of the Lower limb during LT application.

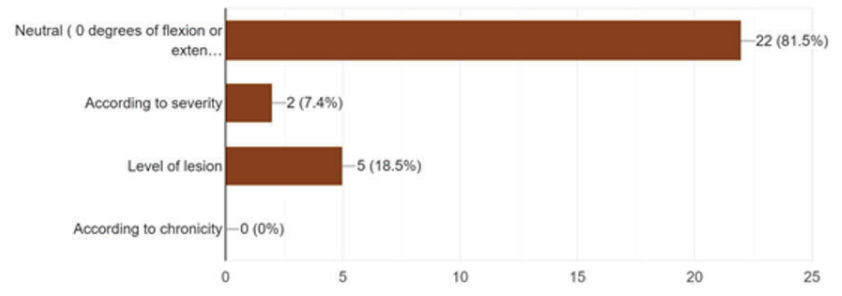

Figure 6: Position of Head during CT application.

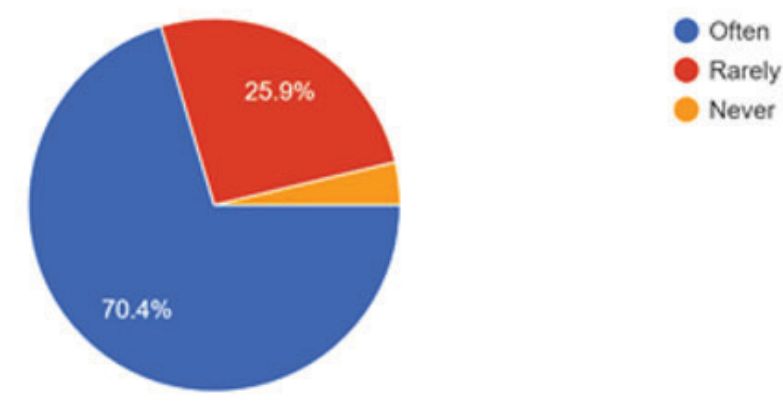

Figure 7: Use of Diathermy in the clinical setup.

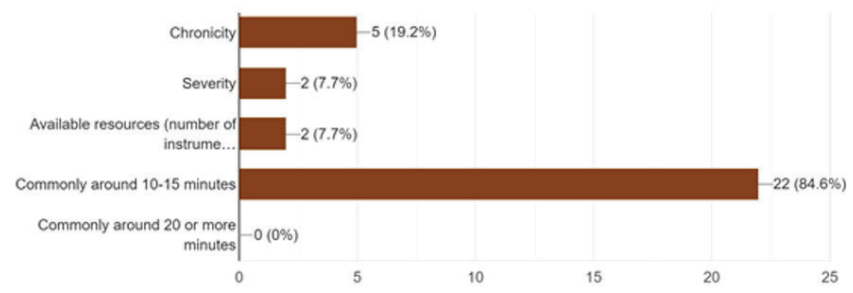

Figure 8: Duration of SWD application.

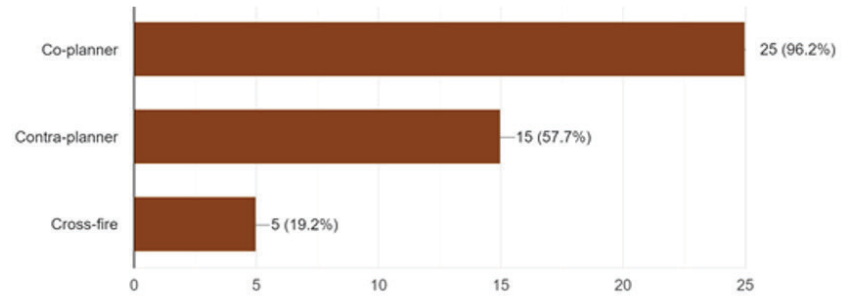

Figure 9: Method of SWD application. 


\section{DISCUSSION}

From the analysis of the data obtained it was observed that all of the institutes were using Ultrasound (US) and Cervical Traction (CT) frequently. Some did not use Short-Wave Diathermy (SWD) or Lumbar Traction (LT). Water bath and water bag were the least preferable methods for the US. The majority is giving the US for 5-7 min, ignoring many aspects in deciding the dosage of it.

Intermittent and Supine with 90-90 position for 10-20 minutes was the most common parameters used for LT. The prone method is almost unused. CT is usually not differentiated with lesion site but given in neutral position. SWD is given according to available resources or in general about 10-15 min in the Co-planner method (Contra-planner and cross-fire being less popular) and rarely as severity or chronicity.

These trends suggest that for US therapy not all methods of application are applied. This may be on account of ease of delivery with Direct contact with Gel method of application.

$\mathrm{CT}$ and LT were also prescribed to the patients irrespective of lesion type. Previously, Phil Harris has concluded that CT is effective only if proper Differential Diagnosis is made and application has been done based on the Differential Diagnosis. ${ }^{8}$

SWD is given with the resources available and not following factors deciding the treatment time.

From the survey, additionally, it was found that UVR is not used in the majority of the clinical setups.

It can be observed that Physiotherapy College and their OPD do not follow the treatment factors and methods described in Literature every time. This also implies that students, who are enrolled under Physiotherapy colleges learn concepts from literature but may apply knowledge based on practical scenarios (as observed and practised in Physiotherapy OPD or clinics), which might create a dilemma.

This is one of a kind survey to find the trends of Electrotherapy Modality usage among Gujarat state.

\section{CONCLUSION}

From the present survey, it was observed that all of the institutes were using Ultrasound and Cervical Traction frequent- ly. The majority of the clinics were giving Ultrasound for 5-7 min, ignoring many aspects in deciding the dosage of it. Cervical Traction is usually not differentiated with lesion site but given in a neutral position. Short-wave Diathermy is given according to available resources or in general about 10-15 min in the Co-planner method.

\section{ACKNOWLEDGEMENT}

The authors acknowledge the immense help received from the scholars whose articles are cited and included in references of this manuscript. The authors are also grateful to authors/editors/publishers of all those articles, journals and books from where the literature for this article has been reviewed and discussed.

\section{Source of Funding: Nil}

\section{Conflict of Interest: Nil}

\section{Individual Author's Contribution:}

Gosai G: Data Collection, Methodology and Referencing,

Dave Y: Data Collection, and Discussion.

Parmar J: Research Design, and Framework for the research. Chhatlani R: Statistical Analysis and Cross-referencing.

\section{REFERENCES}

1. Watson T. The role of electrotherapy in contemporary physiotherapy practice. Man Ther. 000;5(3):132-41.

2. Watson T. Introduction: current concepts and clinical decision making in electrotherapy. In: Watson T, editor. Electrotherapy: evidence-based practice. 12th ed. Edinburgh: Churchill Livingstone/Elsevier; 2008. p. 3-10.

3. Joshi J, Essentials of Orthopaedics and Applied Physiotherapy, Elsevier Publication, $3^{\text {rd }}$ Edition, pg.439-442

4. Joshi J, Essentials of Orthopaedics and Applied Physiotherapy, Elsevier Publication, $3^{\text {rd }}$ Edition, pg. 548-552

5. Khokhar V, Helpline Electrotherapy for Physiotherapists, Bharat Bharati Prakashan, $3^{\text {rd }}$ Edition, pg.77-88

6. Khokhar V, Helpline Electrotherapy for Physiotherapists, Bharat Bharati Prakashan, $3^{\text {rd }}$ Edition, pg.49-62

7. Singh J, Textbook of Electrotherapy, Jaypee Brothers Publication, $2^{\text {nd }}$ Edition, pg.151-162.

8. Phil R. Harris. Cervical Traction: Review of Literature and Treatment Guidelines, Physical Therapy, 5(1) 1977, p. 910-914 